\title{
EFEITO DO TREINAMENTO DE FORÇA NAS VARIÁVEIS \\ CARDIOVASCULARES EM ADOLESCENTES COM SOBREPESO
}

\author{
EFFECT OF STRENGTH TRAINING ON CARDIOVASCULAR VARIABLES \\ IN OVERWEIGHT ADOLESCENTS \\ EFECTO DEL ENTRENAMIENTO DE FUERZA EN LAS VARIABLES CARDIOVASCULARES \\ EN ADOLESCENTES CON SOBREPESO
}

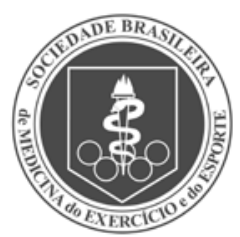

Artigo Original

\begin{abstract}
João Marcelo de Queiroz Miranda',2,4 (Educador Físico)

Leandro da Costa Dias²

(Educador Físico)

Cristiano Teixeira Mostarda ${ }^{3}$

(Educador Físico)

Kátia De Angelis ${ }^{4}$

(Educadora Física)

Aylton José Figueira Junior ${ }^{2}$

(Educador Físico)

Rogério Brandão Wichi ${ }^{5}$

(Educador Físico)

1. Universidade Cidade de São Paulo (Unicid), São Paulo, SP, Brasil. 2. Universidade São Judas Tadeu (USJT), São Paulo, SP, Brasil. 3. Instituto do Coração (InCor, USP), São Paulo, SP, Brasil.

4. Universidade Nove de Julho (Uninove) São Paulo, SP, Brasil.

5. Universidade Federal de Sergipe (UFSE), Aracajú, SE, Brasil.
\end{abstract}

\section{Correspondência:}

Av. 9 de Julho, 1952, apto. 120

São Paulo, SP, Brasil, 01312001

jmtreinamento@yahoo.com.br

\section{RESUMO}

Objetivo: O presente estudo teve por objetivo avaliar as respostas agudas cardiorrespiratórias e autonômicas induzidas por uma sessão de exercício físico resistido em adolescentes com sobrepeso. Métodos: Foram avaliados 17 adolescentes do sexo masculino divididos em grupo controle (GC, n=9) e grupo sobrepeso (GSO, n=7). Todos foram submetidos a uma sessão exercícios resistidos para diferentes grupos musculares, realizados com sobrecarga de 60\% da força máxima, com um intervalo de 45 segundos entre as séries e 90 segundos entre cada exercício. Antes, durante e após a sessão de exercícios os seguintes parâmetros foram avaliados: frequência cardíaca (FC), pressão arterial sistólica (PAS), pressão arterial diastólica (PAD) e variabilidade da frequência cardíaca (VFC). Resultados: Foi observado comportamento semelhante em ambos os grupos da PAS e PAD. A PAS aumentou durante o exercício e a PAD diminuiu. Ambas retornaram aos valores basais durante a recuperação. A FC aumentou durante a sessão de exercício nos dois grupos, porém, apenas no GSO houve retorno aos valores de repouso. Os índices da VFC no domínio do tempo (VAR RR e RMSSD) se comportaram de maneira semelhante frente ao exercício nos dois grupos, porém, no índice SDNN apenas o GSO retornou aos valores de repouso. A VFC no domínio da frequência apresentou comportamento semelhante em ambos os grupos. Conclusões: sugere-se que o aumento do nível de atividade física em indivíduos com sobrepeso pode prevenir as alterações autonômicas que estão associadas ao aumento do peso corporal e conferir efeito protetor ao sistema cardiovascular.

Palavras-chave: treinamento de força, adolescência, obesidade.

\section{ABSTRACT}

Objective: This study aimed to evaluate the acute cardiorespiratory and autonomic responses induced by a resistive exercise session in overweight adolescents. Methods: We evaluated 17 male adolescents divided into control group (CG, $n=9$ ) and overweight group (OWG, $n=7)$. All patients underwent one session of resistance exercises for different muscle groups, performed with overload of $60 \%$ of maximum strength, with an interval of 45 seconds between sets and 90 seconds between each exercise. Before, during and after the exercise session the following parameters were evaluated: heart rate (HR), systolic blood pressure (SBP), diastolic blood pressure $(D B P)$ and heart rate variability (HRV). Results: We observed similar behavior in both groups of SBP and DBP. The SBP increased during exercise while the DBP decreased. Both returned to baseline during recovery. HR increased during the workout in the two groups, but return to resting values only occurred in the OWG. The HRV indices in the time domain (VAR RR and RMSSD) behaved similarly to the exercise in both groups, but the SDNN index only returned to resting values in the OWG. HRV in the frequency domain exhibited similar behavior in both groups. Conclusions: It is suggested that the increased level of physical activity in overweight individuals can prevent autonomic changes that are associated with increased body weight and confer a protective effect on the cardiovascular system

Keywords: strength training, adolescence, obesity.

\section{RESUMEN}

Objetivo: El presente estudio tuvo por objetivo evaluar las respuestas agudas cardiorrespiratorias y autonómicas inducidas por una sesión de ejercicio físico resistido en adolescentes con sobrepeso. Métodos: Fueron evaluados 17 adolescentes del sexo masculino divididos en grupo control (GC, $n=9$ ) y grupo sobrepeso (GSO, $n=7)$. Todos fueron sometidos a una sesión de ejercicios resistidos para diferentes grupos musculares, realizados con sobrecarga de 60\% de la fuerza máxima, con un intervalo de 45 segundos entre las series y 90 segundos entre cada ejercicio. Antes, durante y después de la realización de la sesión de ejercicios los siguientes parámetros fueron evaluados: frecuencia cardiaca (FC), presión arterial sistólica (PAS), presión arterial diastólica (PAD) y 
variabilidad de la frecuencia cardíaca (VFC). Resultados: Fue observado comportamiento semejante en ambos grupos de la PAS y PAD. La PAS aumentó durante el ejercicio y la PAD disminuyó. Ambas retornaron a los valores basales durante la recuperación. La FC aumentó durante la sesión de ejercicio en los dos grupos, no obstante, solamente en el GSO hubo retorno a los valores de reposo. Los índices de la VFC en el dominio del tiempo (VAR RR y RMSSD) se comportaron de manera semejante frente al ejercicio en los dos grupos, no obstante, en el índice SDNN sólo el GSO retornó a los valores de reposo. La VFC en el dominio de la frecuencia presentó comportamiento semejante en ambos grupos. Conclusiones: Se sugiere que el aumento del nivel de actividad física en individuos con sobrepeso puede prevenir las alteraciones autonómicas que están asociadas al aumento del peso corporal y conferirle efecto protector al sistema cardiovascular.

Palabras clave: entrenamiento de fuerza, adolescencia, obesidad.

\section{INTRODUÇÃO}

Estudos epidemiológicos têm considerado a obesidade como uma doença crônica, multifatorial com alta prevalência mundial em países desenvolvidos e em desenvolvimento, caracterizando um quadro epidêmico presente em diferentes grupos etários ${ }^{1,2}$.

Segundo Carneiro ${ }^{2}$ os adolescentes obesos que permanecerem acima do peso na idade adulta estarão sujeitos às complicações neuro-metabólicas e endócrinas, resultando no desenvolvimento das doenças cardiometabólicas precocemente. Sabe-se que indivíduos obesos apresentam disautonomia simpato-vagal, fato associado à atuação no controle cardiovascular pelo sistema nervoso autônomo. Porém, resultados divergentes são encontrados na literatura frente a análise que o comportamento da atividade nervosa simpática encontra-se aumentada 3 ou diminuída em pessoas obesas 4 .

O estudo da variabilidade da frequência cardíaca no domínio do tempo e da frequência, são utilizados como ferramenta investigativa simples e não invasiva direcionada à detecção e análise das disfunções autonômicas cardíacas em diversas patologias, entre elas a obesidade 5 . $O$ estudo no domínio do tempo traz informações a respeito da variabilidade da frequência cardíaca total, estimada através das variações batimento a batimento ${ }^{6}$. Já o estudo do domínio da frequência tem alcançado considerável interesse, por estimar a atividade neural nas oscilações a curto e longo prazo da frequência cardíaca ${ }^{7}$. Assim, consideramos ser relevante a utilização dessa ferramenta durante o exercício físico.

O exercício físico aeróbio parece modular o impacto negativo dos fatores de risco na manifestação das doenças cardiovasculares ${ }^{8-10}$. Por outro lado, algumas evidências tem demonstrado que os exercícios resistidos auxiliam no controle glicêmico e insulinêmico assim como na modulação da pressão arterial ${ }^{11}$ e na redução do peso corporal ${ }^{12}$. Dessa forma, a prática de exercício físico tem sido recomendada como alternativa na prevenção e no tratamento das doenças crônicas, demonstrando melhora de indicadores de saúde e redução das taxas de morbi-mortalidade ${ }^{13}$. Porém, até o presente momento, não foi demonstrado qual o comportamento fisiológico agudo cardiovascular promovido pelo exercício resistido em adolescentes com sobrepeso. Assim, o objetivo deste estudo foi avaliar a resposta aguda autonômica e cardiovascular em adolescentes eutróficos e com sobrepeso submetidos à uma sessão de exercícios resistidos.

\section{MATERIAIS E MÉTODOS}

\section{Casuística}

O projeto de pesquisa foi aprovado pelo Comitê de Ética em Pesquisa - CEP da Universidade São Judas Tadeu (protocolo 007/2007).
Foram selecionados de modo não probabilístico 16 adolescentes do sexo masculino. Os critérios de inclusão foram: ser classificado como pós-púbere pelos critérios de Tanner ${ }^{14}$; ser fisicamente ativo considerando o nível de consumo de $\mathrm{O}_{2}$ acima de $35 \mathrm{ml} / \mathrm{kg} / \mathrm{min}^{15}$. Adolescentes que ingeriam qualquer medicamento ou substância que pudessem comprometer as respostas cardiovasculares e/ou autonômicas foram excluídos da amostra, bem como qualquer restrição médica para execução do exercício resistido. A amostra foi dividida em dois grupos sendo: grupo controle (GC=9) com idade média de

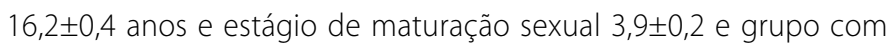
sobrepeso $(\mathrm{GSO}=7)$ com idade média de 16,4 $\pm 0,6$ anos e estágio

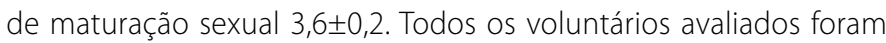
autorizados a participar pelos responsáveis assinando o Termo de Consentimento Livre e Esclarecido.

A avaliação antropométrica consistiu da mensuração do peso corporal $(\mathrm{kg})$ e estatura $(\mathrm{m})$ utilizando um estadiômetro e uma balança de marca Filizola (Brasil). Tais variáveis foram utilizadas no calculo do índice de massa corpórea, IMC $\left(\mathrm{kg} / \mathrm{m}^{2}\right)$. A relação da circunferência cintura-quadril (RCCQ) foi mensurada a partir das suas respectivas circunferências, utilizando uma fita métrica da marca Sanny. As dobras cutâneas da região do tríceps e subescapular foram mensuradas pelo adipômetro da marca Sanny e a composição corporal determinada pelo método da bioimpedância (Modelo Biodynamics, 450) obedecendo aos procedimentos recomendados para o seu uso ${ }^{16}$.

O consumo máximo de oxigênio $\left(\mathrm{VO}_{2 \text { máx }}\right)$ foi determinado pelo teste ergoespirométrico em cicloergômetro modelo CIBEX THE BIKE e sistema metabólico computadorizado (VO2000 Imbrasport). Utilizamos o protocolo de Kawamoto ${ }^{17}$ iniciando o teste com carga de 30 watts incrementando a cada 60s, 15 ou 30 watts de acordo com a massa corporal do sujeito. A velocidade foi mantida constante em $30 \mathrm{Km} / \mathrm{h}$ até que fosse atingida a FC submáxima calculada através da fórmula de Karvonen ${ }^{18}$. A pressão arterial (PA) foi mensurada a cada 3 minutos pelo método auscultatório através do uso de esfignomanômetro aneróide de manguito da marca Missouri ${ }^{\circledR}$ e estetoscópio de marca Littman seguindo as devidas recomendações ${ }^{19}$. Durante o teste, um médico esteve presente monitorando o eletrocardiograma de esforço (ECG). A frequência cardíaca (FC) foi monitorada através da utilização de um frequencímetro da marca Polar ${ }^{\circledR}$ modelo S810.

A VFC foi obtida através do registro do intervalo R-R (IP, ms) pelo frequencímetro da marca Polar ${ }^{\circledR}$ modelo 5810 . Os arquivos de registro foram transferidos para o Polar Precision Performance Software permitindo a troca de dados do exercício para o computador para posterior análise. Após aquisição e armazenamento dos dados, os intervalos R-R foram convertidos e armazenados em arquivos Excel e utilizados posteriormente na análise da VFC. As variâncias das séries de IP foram 
avaliadas no domínio do tempo e no domínio da frequência ${ }^{20}$. Utilizamos o software HRV Analisys para mensurar cada uma das variáveis da VFC em todos os sujeitos.

Três dias antes da realização da sessão de exercícios resistidos, cada participante foi submetido a um teste de uma repetição máxima (1RM) nos exercícios utilizados no protocolo. Cada exercício foi precedido de um aquecimento (6 a 10 repetições) com 50\% da carga registrada na primeira tentativa do 1RM. Três tentativas com intervalos de 3 a 5 minutos entre elas foram dadas aos participantes. Todos os participantes foram familiarizados ${ }^{21}$ aos exercícios nas sessões realizadas nos dias anteriores.

\section{Protocolo de treinamento}

A sessão de exercício resistido foi realizada quarenta e oito horas após o teste de uma repetição máxima. O protocolo de treinamento foi composto de cinco exercícios: supino vertical (SUP), leg-press (LEG), puxador no pulley (PUL), cadeira extensora (CAE) e rosca direta (RD), realizados com 60\% de 1RM com três séries de cada exercício com 15 repetições cada série e intervalo de 45 segundos entre as séries e 90 segundos entre exercícios ${ }^{22}$ totalizando trinta minutos de exercícios. Dez minutos antes do início da sessão de treinamento, durante os intervalos entre os exercícios e 30 minutos após a sessão foram mensuradas e FC e VFC, enquanto que a PA foi mensurada antes e após a sessão de exercícios.

\section{Análise dos dados}

Os resultados foram analisados pelo teste de Kolmogorov e Smirnov para verificar a distribuição da amostra. Foi aplicado o teste $t$ de student pareado na comparação entre os grupos GC e GSO. A análise de variância (ANOVA) de dois fatores para medidas repetidas seguido do teste de Newman Keuls post-hoc foram aplicados na análise resultados da sessão de exercício resistido, sendo o nível de significância de $p<0,05$.

\section{RESULTADOS}

Os dados do presente estudo demonstraram que as variáveis analisadas comportaram-se de maneira semelhante em ambos os grupos. De modo geral, a PAS, FC e atividade simpática aumentaram ao longo da sessão, retornando aos seus valores de repouso durante o período de recuperação.

Na tabela 1 são demonstrados os resultados antropométricos e composição corporal do GC e GSO. Encontramos similaridade na idade, estágio maturacional, estatura e nível de atividade física em ambos os grupos. Por outro lado, o GSO apresentou valores superiores de peso corporal, IMC, circunferência da cintura e quadril $(p<0,05)$ em relação ao GC. A análise da composição corporal evidenciou que o GSO apresentou maior valor absoluto de massa magra (Kg) que o GC. Porem em valor relativo (\%) observou-se tendência inversa, tendo o GC apresentado maior contribuição da massa muscular na composição corporal. O GSO apresentou maior adiposidade que o GC.

A análise das variáveis cardiorrespiratórias evidenciou que o GSO apresentou maiores valores na FC de repouso e maior valor no consumo de oxigênio ( $L / \mathrm{min})$, não sendo encontradas diferenças nos valores de PAS e PAD entre os grupos (tabela 2).

A comparação dos valores da força máxima segue na tabela 3, demonstrando similaridade entre os grupos GC e GSO nos cincos exercícios propostos.

Na figura 1 é apresentado o comportamento das variáveis cardiovasculares antes, durante e após a sessão de exercícios. A PAS demonstrou elevação significativa em relação ao repouso a partir do
TABELA 1. Comparação das características antropométricas e composição corporal entre adolescentes eutróficos (GC) e com sobrepeso (GSO).

\begin{tabular}{|c|c|c|}
\hline Variáveis & GC & GSO \\
\hline Peso Corporal (Kg) & $63,6 \pm 1,7$ & $82,2 \pm 1,6^{*}$ \\
\hline Altura (m) & $1,7 \pm 0$ & $1,7 \pm 0$ \\
\hline $\operatorname{IMC}\left(\mathrm{Kg} / \mathrm{m}^{2}\right)$ & $21,9 \pm 0,6$ & $27,2 \pm 0,4^{*}$ \\
\hline Massa Magra (Kg) & $55,2 \pm 1,5$ & $61,5 \pm 2,5^{*}$ \\
\hline Massa Magra (\%) & $86,5 \pm 1,4$ & $74,7 \pm 2,1$ \\
\hline Massa Gorda (Kg) & $8,6 \pm 1$ & $20,7 \pm 1,5^{*}$ \\
\hline Massa Gorda (\%) & $13,4 \pm 1,4$ & $25,3 \pm 2,1^{*}$ \\
\hline Circunferência da Cintura (cm) & $67,9 \pm 0,7$ & $83,8 \pm 1,8^{*}$ \\
\hline Circunferência do Quadril (cm) & $88 \pm 0,6$ & $96,9 \pm 0,7^{*}$ \\
\hline Relação Cintura / Quadril & $0,77 \pm 0,01$ & $0,87 \pm 0,02^{*}$ \\
\hline $\mathrm{DOC} T R(\mathrm{~mm})$ & $10,6 \pm 1,1$ & $17,4 \pm 0,7^{*}$ \\
\hline DOC SE (mm) & $9,7 \pm 0,5$ & $10,2 \pm 2,3$ \\
\hline
\end{tabular}

Os valores representam média \pm desvio padrão; * $p<0,05$ em relação ao grupo. controle (GC).

Tabela 2. Comparação de indicadores cardiorrespiratórios entre adolescentes eutróficos (GC) e com sobrepeso (GSO).

\begin{tabular}{c|c|c}
\hline Variáveis & GC & GSO \\
\hline Pressão Arterial Sistólica $(\mathrm{mmHg})$ & $108 \pm 4,1$ & $116 \pm 3$ \\
\hline Pressão Arterial Diastólica $(\mathrm{mmHg})$ & $71,3 \pm 2,3$ & $73,7 \pm 1,2$ \\
\hline Frequência Cardíaca de Repouso $(\mathrm{bpm})$ & $67,2 \pm 1,7$ & $73,4 \pm 2,2^{*}$ \\
\hline Consumo Máximo de Oxigênio $(\mathrm{L} / \mathrm{min})$ & $3,6 \pm 0,1$ & $4,6 \pm 0,1^{*}$ \\
\hline Consumo Máximo de Oxigênio $(\mathrm{mL} / \mathrm{Kg} / \mathrm{mim})$ & $59,6 \pm 0,6$ & $56,1 \pm 0,6$ \\
\hline
\end{tabular}

Os valores representam média \pm desvio padrão; * $\mathrm{p}<0,0505$ em relação ao grupo controle (GC).

Tabela 3. Carga obtida no teste de 1RM em diferentes exercícios em adolescentes eutróficos (GC) e com sobrepeso (GSO).

\begin{tabular}{c|c|c}
\hline Variáveis & GC & GSO \\
\hline Supino Vertical $(\mathrm{kg})$ & $50 \pm 4$ & $49 \pm 11$ \\
\hline Leg Press $(\mathrm{kg})$ & $86 \pm 4$ & $77 \pm 16$ \\
\hline Puxador Pulley $(\mathrm{kg})$ & $63 \pm 6$ & $57 \pm 11$ \\
\hline Cadeira Extensora (kg) & $68 \pm 9$ & $70 \pm 18$ \\
\hline Rosca Direta $(\mathrm{kg})$ & $29 \pm 2$ & $27 \pm 3$ \\
\hline
\end{tabular}

Os valores médios \pm desvio padrăo; ${ }^{*} \mathrm{p}<0,0505$ em relaçăo ao grupo controle (GC). 
segundo exercício tanto no GC quanto no GSO, sendo que na fase de recuperação, ambos decresceram ao nível de repouso. Não foi observada diferença entre os grupos nos cinco exercícios propostos (figura 1A). Por outro lado, o comportamento da PAD apresentou diminuição significante no GC após o quarto exercício, retornando aos valores iniciais na fase de recuperação, assim como observado no GSO. Não foi observada diferença entre os grupos durante os exercícios e na recuperação (figura $1 \mathrm{~B}$ ).

A figura $1 \mathrm{C}$ demonstra o comportamento da FC. Encontramos que o GC apresentou aumento significativo a partir do segundo exercício, sendo que no período de recuperação a FC permaneceu com valores

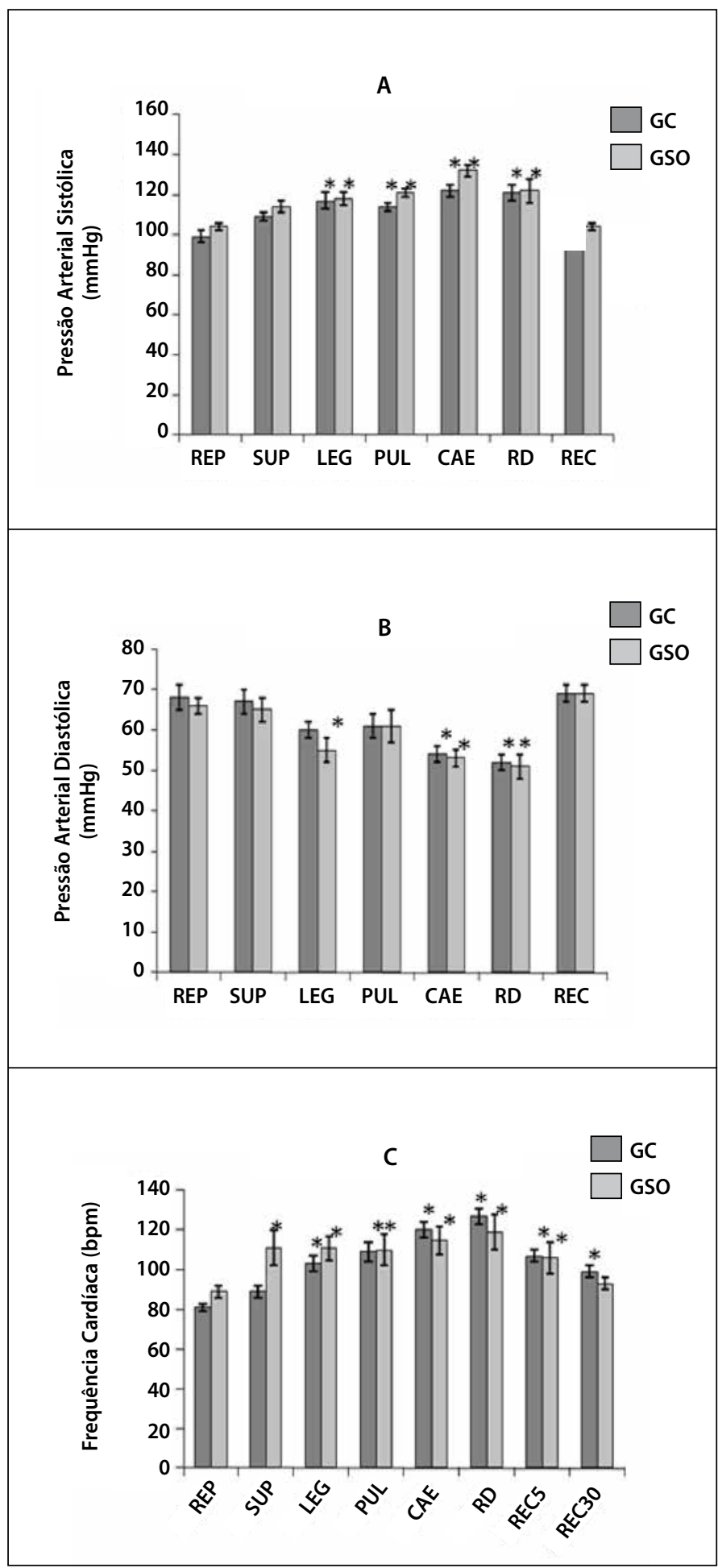

Figura 1. Comportamento das variáveis cardiovasculares em adolescentes eutróficos e com sobrepeso antes, durante, e depois de uma sessão de exercícios resistidos. acima do repouso todo o período pós-esforço. No GSO, observou-se aumento da FC em relação ao repouso e a partir do primeiro exercício, porém, diferentemente do GC, a FC retornou aos valores de repouso em 30 minutos de recuperação.

As variáveis autonômicas são apresentadas na tabela 4. No índice de domínio do tempo (VAR RR, SDNN e RMSSD) foi observado em ambos os grupos redução no período de recuperação em 5 e 30 minutos com relação ao valor basal $(p<0,05)$, demonstrando comportamento similar nessas variáveis. Já a análise da VFC no domínio da frequência, (HF, LF e LF/HF) demonstrou que, em ambos os grupos, não foram verificadas diferenças significativas entre os grupos nos momentos de recuperação (Rec5 e Rec30) em relação ao momento de repouso.

Tabela 4. Variabilidade da frequência cardíaca no domínio do tempo e da frequência nos grupos controle e sobrepeso.

\begin{tabular}{c|c|c|c|c|c|c}
\hline \multirow{2}{*}{ Variáveis } & \multicolumn{3}{c|}{ GC } & \multicolumn{3}{c}{ GSO } \\
\hline & Rep & $\operatorname{Rec5}$ & $\operatorname{Rec30}$ & $\operatorname{Rep}$ & $\operatorname{Rec5}$ & $\operatorname{Rec30}$ \\
\hline VAR RR (ms) & $2524 \pm 438$ & $422 \pm 101^{*}$ & $926 \pm 144^{*}$ & $2523 \pm 481$ & $939 \pm 284^{*}$ & $1220 \pm 311^{*}$ \\
\hline SDNN (ms) & $56 \pm 8$ & $19 \pm 2^{*}$ & $29 \pm 2^{*}$ & $48 \pm 5$ & $28 \pm 5^{*}$ & $32 \pm 5$ \\
\hline RMSSD (ms) & $45 \pm 9$ & $13 \pm 1^{*}$ & $15 \pm 1^{*}$ & $43 \pm 7$ & $20 \pm 4^{*}$ & $23 \pm 5^{*}$ \\
\hline HF (n.u) & $26 \pm 3$ & $21 \pm 4$ & $20 \pm 4$ & $33 \pm 6$ & $20 \pm 3$ & $18 \pm 3$ \\
\hline LF (n.u) & $73 \pm 3$ & $79 \pm 4$ & $80 \pm 4$ & $66 \pm 6$ & $79 \pm 3$ & $82 \pm 3$ \\
\hline LF/HF & $3,6 \pm 1$ & $5,7 \pm 2$ & $4,3 \pm 1$ & $3,2 \pm 1$ & $3,1 \pm 0$ & $4,1 \pm 1$ \\
\hline
\end{tabular}

Os valores representam média \pm erro padrão; ${ }^{*} \mathrm{p}<0,05$ Rep vs. Rec5; Rep vs. Rec30.

\section{DISCUSSÃO}

No presente estudo, encontramos similaridade nas idades, estágio maturacional, potência aeróbia e força muscular. Guízar et al., ${ }^{23}$ avaliaram o estágio maturacional pelos critérios de Tanner ${ }^{14}$ em setenta adolescentes do sexo masculino, demonstrando que o grupo com sobrepeso não apresentou diferença no estágio maturacional quando comparado ao grupo controle. No presente estudo foi encontrada semelhança no estágio maturacional entres os grupos.

Por outro lado diferenças foram observadas nas características antropométricas e da composição corporal entre CG e GSO. Em um estudo realizado por Carneiro 2 foi demonstrado o estado nutricional e a relação da circunferência cintura-quadril em obesos e não obesos, onde - IMC e RCQ foram maiores no grupo de obesos similar ao encontrado no presente estudo. Em outro estudo, Rodrigues et al. ${ }^{24}$ avaliaram a aptidão cardiorrespiratória em adolescentes de ambos os sexos. Os dados evidenciaram que os meninos com a aptidão cardiorrespiratória fraca possuíam maior IMC e maior FC basal quando comparado ao grupo com maior aptidão cardiorrespiratória, sugerindo influencia negativa da adiposidade. Em outro estudo, Ribeiro et al., ${ }^{25}$ avaliaram 
o consumo pico de oxigênio em crianças obesas e não obesas e observaram que as crianças obesas apresentavam menores valores que as crianças com peso normal. Ainda, Norman et al., ${ }^{26}$ utilizaram o cicloergômetro para avaliar o consumo pico de oxigênio de adolescentes com sobrepeso. Foi encontrado que o grupo com sobrepeso apresentou $\mathrm{VO}_{2}$ pico menor que o grupo controle, No nosso estudo não foram encontrados diferenças entre os grupos nessa variável. Imaginamos que esses dados sejam explicados pelo fato dos adolescentes no presente estudo serem fisicamente ativos. Porém, o GC apresentou menor $\mathrm{VO}_{2}$ comparado ao GSO quando consideramos o valor absoluto do $\mathrm{VO}_{2 \text { máx }}(\mathrm{L} / \mathrm{min})$.

Além disso, no presente estudo, os grupos apresentaram valores semelhantes de pressão arterial sistólica (PAS) e diastólica (PAD). Os valores encontrados classificam a nossa amostra com níveis pressóricos normais ${ }^{19}$. Estudo de Carletti et al. ${ }^{27}$ demonstrou aumento na PAS em repouso em meninos com sobrepeso e obesidade quando comparados a meninos normais. Esta diferença pode ser atribuída ao grau de obesidade encontrado no estudo. Em estudo transversal realizado por Marcos et al. ${ }^{28}$ com adolescentes mexicanos não-obesos e obesos, os autores encontraram maior valor na PAS e PAD no grupo dos obeso. Os níveis pressóricos aumentados foram associados à adiposidade central e podem ser explicados pela hiperinsulinemia encontrada na amostra, afetando o sistema nervoso simpático e a função endotelial.

Por outro lado, durante o exercício físico, as respostas cardiovasculares e autonômicas parecem se comportar distintamente entre indivíduos com sobrepeso e normais. Carletti et al. ${ }^{27}$ avaliaram o comportamento da PA em adolescentes de ambos os sexos, normotensos, com peso normal e sobrepeso. Os meninos com sobrepeso apresentaram maiores valores de PAD antes e após realizarem exercício máximo em esteira. Na PAD não foi observado diferenças entre os grupos nem antes do exercício e nem após o pico de exercício. Este comportamento não foi observado na PAS de nosso estudo, porém, resultados semelhantes foram encontrados na PAD. Podemos explicar tais diferenças pelo tipo de exercício utilizado, intensidade, volumes e nível de atividade física ser diferentes entre os grupos. Os autores do estudo sugerem que tais resultados sejam atribuídos à atividade simpática exacerbada no grupo de sobrepeso e obesidade. Em outro estudo Mediano et al. ${ }^{11}$ avaliaram a resposta da pressão arterial aguda em adultos hipertensos submetidos à exercício resistido com diferentes volumes de treinamento. Os sujeitos foram divididos em grupos que realizavam uma série (SER1) ou três séries (SER3) com 10 repetições máximas. A PA foi avaliada nos momentos pré, pós e durante 60 minutos de recuperação. Foi observado aumento da PAS e PAD em ambos os grupos no momento pós-exercício. O nosso estudo encontrou tendência semelhante na PAS, ocorrendo o oposto na PAD, que no presente diminuiu após realização do último exercício. Na recuperação o SER3 apresentou efeito hipotensor da PAS durante os 60 minutos e da PAD apenas no trigésimo e quinquagésimo minutos. Para Mediano et al., ${ }^{11}$ a elevação da pressão arterial ocorreu pela ativação de quimiorreceptores por fadiga periférica, e seu declínio subsequente ao mecanismo barorreflexo, pela hiperemia decorrente da contração muscular e supressão da atividade simpática em decorrência ao exercício. O efeito hipotensor ainda não está bem esclarecido na literatura, porém, acredita-se que a maior liberação de óxido nítrico e menor descarga adrenérgica possam contribuir para tal fenômeno, que segundo Mediano et al., ${ }^{11}$ pode ser influenciado pelo maior volume de treinamento.
Rezk et al.22 avaliaram a resposta hemodinâmica e autonômica em normotensos jovens antes e após sessões de exercício resistido com intensidades de 40\% e 80\% de 1RM. Na recuperação, a PAS sofreu decréscimo similar em ambos os grupos em relação ao repouso. A PAD diminuiu apenas no grupo que se exercitou a $40 \%$ de 1RM. Apesar de o nosso protocolo experimental ser bastante similar ao utilizado pelo estudo anterior, no presente estudo não foi observado tal comportamento da PA em ambos os grupos. Porém, no estudo citado, ${ }^{22}$ os sujeitos foram acompanhados por 90 minutos de recuperação, enquanto no presente estudo o foram em 30 minutos, além da intensidade em 60\% de 1RM. Tais achados podem ter sido influenciados pela diminuição do débito cardíaco, que também foi mensurado compensando o aumento da resistência vascular sistêmica22.

Em relação ao comportamento da FC durante o exercício resistido, encontramos similaridade nas variáveis estudadas em ambos os grupos. Rezk et al. ${ }^{22}$ encontraram aumento da FC nos grupos experimentais, porém o maior aumento ocorreu no grupo que se exercitou com maior intensidade (80\% de 1RM) até os 30 minutos de recuperação. Comportamento similar da FC também foi observado após exercício no GC. O GSO apresentou aumento da FC em relação ao repouso nos primeiros 5 minutos de recuperação, mas ao final da recuperação de 30 minutos, os valores retornaram aos basais. Tal comportamento da FC é resultante da ativação da atividade simpática e retirada vagal ${ }^{22}$

No presente estudo, os índices de VFC (VAR RR, RMSSD) se comportaram de forma semelhante em ambos os grupos em todos os momentos pesquisados. Em repouso, Guízar et al. ${ }^{23}$ avaliaram a atividade nervosa simpática em adolescentes não obesos e obesos pelo mesmo método do nosso estudo. O grupo de obesos apresentou menor VAR RR, menor SDNN e maior balanço simpato-vagal. Estes autores correlacionaram positivamente o componente LF/HF com o IMC, a RCQ, níveis de leptina e níveis de pressão arterial sistólica. Rezk et al., ${ }^{22}$ verificaram aumento da atividade simpática, diminuição da atividade vagal e maior do balanço simpato-vagal após sessões de exercícios resistido. Tal explicação pode ser atribuída ao aumento da resistência vascular sistêmica que desativa receptores cardiopulmonares aumentando a atividade simpática. Kim et al., ${ }^{29}$ observaram o comportamento autonômico homens e mulheres adultos e obesos no repouso e provocando algum tipo de estímulo, por 5 minutos. Não foram observadas diferenças entre o grupo controle e o grupo de obesos durante os momentos de aquisição da VFC. Porém, o percentual de gordura corporal e a RCQ se correlacionaram negativamente com os índices SDNN, RMSSD e LF. Os autores sugerem que a obesidade possa estar associada aos distúrbios nas atividades simpáticas e parassimpáticas e que os estímulos utilizados não foram suficientes para modificar a VFC quando comparado ao repouso.

Poucos são os estudos científicos que analisaram as variáveis cardiovasculares e autonômicas antes, durante, e após o exercício resistido em adolescentes com excesso de peso. Porém, apontamos no presente estudo alguns fatores limitantes como o tamanho da amostra, pois há dificuldade de se encontrar sujeitos adolescentes obesos e fisicamente ativos que possam participar do estudo, assim como a padronização do consumo alimentar antes da sessão de exercício. Entretanto, ressaltamos que as repostas cardiovasculares e autonômicas foram semelhantes em GC e GSO. Podemos sugerir que o maior percentual de gordura corporal no GSO não influenciou as variáveis quando comparados 
ao GC. Além disso, apontamos que tais resultados podem estar relacionados com o nível de atividade física que pode ser compensado, protegendo dos efeitos deletérios do excesso de peso no comportamento cardiovascular.

\section{CONCLUSÃO}

A partir dos dados do presente estudo, podemos concluir que no inicio de um programa de exercício resistido haverá elevação da FC e da PAS, acompanhados da diminuição da VAR RR e da atividade vagal (RMSSD) em sujeitos fisicamente ativos.

Todos os autores declararam não haver qualquer potencial conflito de interesses referente a este artigo.

\section{REFERÊNCIAS}

1. Oliveira CS, Veiga GV. Estado Nutricional e Maturação Sexual de Adolescentes de uma Escola Pública e de uma Escola Privada do Município do Rio de Janeiro. Rev Nut.2005;18(2):183-91.

2. Carneiro JRI, Kushnir MC, Clemente ELS, Brandão MG, Gomes MB. Obesidade na Adolescência: Fator de Risco para Complicações Clínico-Metabólicas. Arq. Bras. Endocrinol. Metab.2005;44 (5):390-6.

3. Grassi G. Debating sympathetic overactivity as a hallmark of human obesity: a pro's position. J.Hypertens. 1999;17:1059-106.

4. Laederach-Hofmann, K; Mussgay, L; Ruddel, H. Autonomic cardiovascular regulation in obesity. J En docrinol..2000;Jan;164(1):59-66.

5. Montano N. Heart rate variability as a clinical tool. Ital Heart J .2002;3:439.

6. Task Force of the European Society of Cardiology and the North American Society of Pacing and Electrophysiology. Heart rate variability: standards of measurements, physiological interpretation and clinical use. Circulation. 1996;93:1043-65.

7. De Angelis K, Santos MSB, Irigoyen MC. Sistema nervoso autônomo e doença cardiovascular. Revista da Sociedade de Cardiologia do Rio Grande do Sul.2004;3.

8. Durstine JL, Haskell WL. Effects of exercise training on plasma lipids and lipoproteins. Exerc Sport Sci Rev.1994;22:477-521.

9. Barbato KBG, Martins RCV, Rodrigues MLG, Braga JU, Francischetti EA, Genelhu V. Efeitos da redução de peso superior a $5 \%$ nos perfis hemodinâmico, metabólico e neuroendócrino de obesos grau 1.Arq Bras Cardiol. 2006;87(1):12-21.

10. Paffenbarger RS, Jung DL, Leung RW, Hyde RT. Physical activity and hypertension: an epidemiological view. Ann Med. 1991;23:319-27.

11. Mediano MFF, Paravidino V, Simão R, Polito MD, Pontes FL. Comportamento subagudo da pres são arterial após o treinamento de força em hipertensos controlados. Rev bras med Esporte. 2005;11(6):337-40.

12. Hauser C, Benetti M, Rebelo FPV. Estratégias para o emagrecimento. Rev. Bras. Cineantropom. Des empenho Hum. 2004;6(1):72-81.

13. I-Min Lee, Eric J Shiroma, Felipe Lobelo, Pekka Puska, Steven N Blair, Peter T Katzmarzyk, Effect of physical inactivity on major non-communicable diseases worldwide: an analysis of burden of disease and life expectancy. Lancet. 2012;380(9838):219-29 http://dx.doi.org/10.1016/S01406736(12)61031-9

14. Tanner JN. Growth at adolescence with a general consideration of the effects of hereditary and environmental factors upon growth and maturation from birth to maturity. 2nd ed. Oxford: Blackwell Scientific Publications; 1962.
15. Pitanga FJG. Testes, Medidas e Avaliação em Educação Física e Esportes. Phorte Editora, ed 4, São Paulo, 2005 16. Giannichi RS, Rigueira JE, Bedim RF. Análise da técnica de bioimpedância elétrica em relação a sua validade. R. Min. Educ. Fís., Viçosa, v. 8, n. 2, p. 7-2 1, 2000.

17. Kawamoto RU. Efeitos do exercício físico agudo contínuo e intermitente sobre o gasto energético: comparação entre adolescentes obesos e eutróficos. Dissertação (Mestrado em Educação Física) Universidade São Judas Tadeu, São Paulo, 2007

18. Karvonen M.J., Kentala E., Mustala O. The effects of training on heart rate; a longitudinal study. Ann Med Exp Biol Fenn.1957;35(3): 307-15.

19. American College of sports medicine. Diretrizes do ACSM para os testes de esforço e sua prescrição. 6. ed. Rio de Janeiro: Guanabara Koogan, 2003.

20. Malliani A, Pagani M, Lombardi F, Cerutti S. Cardiovascular neural regulation explored in the frequency domain. Circulation. 1991;84:482-492.

21. Dias RMR, Cyrino ES, Salvador EP, Caldeira LFS, Nakamura FY, Papast RR, Bruna N, Gurião ALD. Influência do processo de familiarização para avaliação da força muscular em testes de 1-RM. Rev Bras Med Esporte. 2005;11(1):34-8.

22. RezkCC,Marrache RCB,TinucciT,Mion JrD, Forjaz CLM. Post-resistance exercise hypotension,hemodynamics,and heart rate variability:influence of exercise intensity. Eur. J. Appl. Physiol. 2006;98(1):105-12.

23. Guízar JM, Ahuatzin R, Amador N, Sánchez G, Romer G. Heart Autonomic Function in Overweight Adolescents. Indian Pediatrics. 2005;42(17),

24. Rodrigues AN, Perez AJ, Carletti L, Bissoli NS, Abreu GR. The association between cardiorespiratory fitness and cardiovascular risk in adolescents. J Pediatr. 2007;83(5):429-35.

25. Ribeiro RQC, Lotufo PA, Lamounier JA, Oiveira RG, Soares JF, Botter DA. Fatores adicionais de risco cardiovascular associados ao excesso de peso em crianças e adolescentes. O estudo do coração de Belo Horizonte. Arq. Bras. Cardiol. 2006;86(6):408-18.

26. Norman AC, Drinkard B, McDuffie JR, Ghorbani S, Yanoff LB, Yanovski JA. Influence of Excess Adiposity on Exercise Fitness and Performance in Overweight Children and Adolescents. Pediatrics. 2005;115(6):e690-e696.

27. Carletti L, Rodrigues AN, Perez AJ, Vassallo DV. Resposta da Pressão Arterial ao Esforço em Adolescentes: Influência do Sobrepeso e Obesidade. Arq Bras Cardiol. 2008;91 (1):25-30

28. Marcos NJD, Núñez GMR, Salinas AMM, Santos MA, Decanini HA. Obesidad como Factor de Riesgo para Trastornos Metabólicos en Adolescentes Mexicanos.Rev. Salud Publica. 2007;9 (2):180-93.

29. Kim JA, Park YG, Cho KH, Hong MH, Han HC, Choi YS, Yoon D. Heart Rate Variability and Obesity Indices: Emphasis on the Response to Noise and Standing. J Am Board Fam Pract.2005;18:97-103. 